\section{$\mathrm{BPH}$ : grünes Licht für die Prostata}

$\mathrm{W}^{\mathrm{i}}$ ie PD Dr. Matthias Oelke, Hannover, bemängelte, seien benigne Prostataerkrankungen - gemessen an ihrer Häufigkeit und volkswirtschaftlichen Bedeutung - auf Kongressen leider in letzter Zeit eher unterrepräsentiert. Nur 3,5\% aller eingereichten Abstracts beschäftigen sich mit diesem Krankheitsbild, das an siebter Stelle der häufigsten und teuersten Krankheiten steht [Fenter et al. Amer J Manag Care 2006; 12: 90-8].

Beim DGU-Kongress wurde nun von Studien zur benignen Prostatahyperplasie (BPH) berichtet. So würde unter anderem über Alternativen zur transurethralen Resektion der Prostata (TURP) referiert. In den letzten Jahren hat sich auch die Vaporisation des Gewebes mit dem Greenlight-Laser immer mehr durchgesetzt. Durch die Laserenergie wird nicht nur das Gewebe schonend entfernt, sondern auch die Blutgefäße werden so effektiv verschlossen, dass dieses Verfahren auch bei Patienten angewendet werden kann, die blutverdünnende Medikamente einnehmen. Der Krankenhausaufenthalt kann unter diesen Voraussetzungen in der Regel auf wenige Tage reduziert werden. Bisher waren hauptsächlich 80- und 120-Watt-Greenlight-Laser im Einsatz. Die Arbeitsgruppe von M. Rieken aus Basel untersuchte nun, ob der neue, leistungsstärkere 180-Watt-GreenLightLaser mehr Gewebe bei gleicher Morbidität verdampfen kann. In diese Kohortenuntersuchung wurden jeweils 67 Patienten entweder mit dem 180-Watt-Gerät oder den schwächeren Lasern behandelt. Es zeigte sich, dass mit dem neuen Greenlight-Laser eine erhöhte Gewebeablation bei gleichbleibender Sicherheit möglich ist.

Bettina Reich

\section{Enuresis: erfolgreiche Urotherapie}

B ei nächtlichem Einnässen im Kindesalter kann häufig eine Urotherapie - das heißt eine Verhaltensumstellung - hilfreich sein, wie Dr. Brigitte Willer, Villingen-Schwenningen, erklärte. $\mathrm{Zu}$ Beginn muss jedoch eine diagnostische Abgrenzung erfolgen. Dazu sollte das rein nächtliche Einnässen (monosymptomatische Enuresis nocturna; MEN) von einem nächtlichen Einnässen mit zusätzlichen Tagessymptomen (nicht monosymptomatische Enuresis nocturna; Non-MEN) und dem isolierten Einnässen am Tag unterschieden werden.

Im Kontinenzzentrum Südwest in Villingen-Schwennigen wurden zwischen 2005 und 2008140 Kinder mit einer monosymptomatischen Enuresis behandelt und in eine vergleichende Analyse zwischen Urotherapie und medikamentöser Behandlung mit Vasopressin einbezogen. $45 \%$ der Kinder nahmen an einer Urotherapie teil. Die Mehrheit der restlichen Patienten bekam Medikamente. Im Rahmen der Urotherapie wurde zuerst ein Miktionsprotokoll erarbeitet. „Ganz deutlich zeigte sich, dass abends am meisten getrunken wird und dass die Kinder auch im Laufe des Tages nicht häufig genug auf die Toilette gehen“, berichtete Willer. Daher wurde ein Trink- und Miktionsplan erstellt. Die Kinder sollten $90 \%$ der Trinkmenge bis 17 Uhr zu sich nehmen. Die Erfolgskontrolle wurde in einem Einnäss-Kalender notiert.

Unter der konsequent durchgeführten Urotherapie wurden $70 \%$ der Kinder komplett trocken. Nur bei $5 \%$ zeigte sich kein Erfolg, da sie die Verhaltenstherapie nicht befolgt hatten. In der Gruppe der Kinder, die Desmopressin bekommen hatten, wurden $52 \%$ komplett trocken.

Bettina Reich

\title{
Urologen arbeiten NS-Vergangenheit auf
}

D ie DGU hat sich die Aufarbeitung des Nationalsozialismus für ihr Fachgebiet schon seit Langem zur Aufgabe gemacht. In einem von der DGU initiierten Forschungsprojekt haben $\mathrm{Me}$ dizinhistoriker und Urologen aus Deutschland, Österreich und den USA in den letzten dreieinhalb Jahren viele bislang unberührte Themen der NS-Zeit bearbeitet und jetzt in einem Buch zusammengefasst. Mitherausgeber Prof. Dr. Dirk Schultheiss, Gießen, Archivar der DGU, stellte den Doppelband „Urologen im Nationalsozialismus. Zwischen Anpassung und Vertreibung “im Rahmen des diesjährigen DGU-Kongresses vor. „Wir haben das Buch auf die Personen bezogen, schon allein im Titel“, kommentierte Schultheiss die Inhalte. Einzel- und Sammelbiografien dokumentieren so- wohl die Schicksale der Opfer als auch die Werdegänge derer, die in dem System Karriere machten. Weitere Themen: die Geschichte der Fachgesellschaft während und nach der Nazidiktatur, der Umgang der beiden deutschen Staaten mit der Vergangenheit und fachhistorische Aspekte wie zum Beispiel die Beiträge von Urologen zur Zwangssterilisation sowie die Entwicklung der Geschlechtskrankenfürsorge, der Sexualmedizin und der Andrologie. Nach Worten von Schultheiss war vor der nationalsozialistischen Machtübernahme 1933 etwa jeder dritte Urologe in Deutschland jüdischen Glaubens oder jüdischer Herkunft - ein Anteil der im Vergleich zur allgemeinen Ärzteschaft doppelt so hoch ist.

DGU und Herausgeber sehen die Veröffentlichung nicht als Abschluss des Pro- jekts zur Aufarbeitung der Vergangenheit, sondern als Ausgangspunkt für eine breitere Diskussion und weitere Forschungsarbeiten. Dr. Claudia Mäck

Eröffnungs-Pressekonferenz

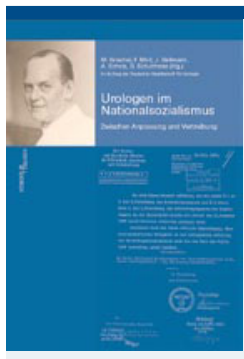

Buchtipp

M. Krische, F. Moll, J. Bellmann, A. Scholz, D Schultheiss. Urologen im Nationalsozialismus. Zwischen Anpassung und Vertreibung Hentrich \& Hentrich, Berlin. Hardcover, je. ca. 300 Seiten. Doppelband 89 EUR (ISBN 978-3-942271-41-7), einzeln 49,90 EUR 